\title{
Simulation of 3D inclined/rotated UV lithography and its application to microneedles
}

\author{
Shijie Liu ${ }^{\mathrm{a}, *}$, Georg Roeder ${ }^{\mathrm{a}}$, Gulnur Aygun ${ }^{\mathrm{b}}$, Kristian Motzek ${ }^{\mathrm{a}}$, Peter Evanschitzky ${ }^{\mathrm{a}}$, Andreas Erdmann ${ }^{\mathrm{a}}$ \\ ${ }^{a}$ Fraunhofer Institute for Integrated Systems and Device Technology (IISB), Schottkystrasse 10, 91058 Erlangen, Germany \\ b Physics Department, Izmir Institute of Technology, 35430, Urla, Izmir, Turkey
}

\section{A R T I C L E I N F O}

\section{Article history:}

Received 14 January 2011

Accepted 17 June 2011

\section{Keywords:}

UV lithography

Inclined and rotated exposure

3D simulation

Microneedles

\begin{abstract}
A B S T R A C T
A 3D model is set up to simulate the exposure process of inclined/rotated UV lithography for negative SU-8 resists. The formation of inclined resist pillars and microstructures with truncated cone shapes is simulated based on a 3D exposure model in combination with a post exposure bake model for chemically amplified resists and the Mack development model. As one of the interesting applications employing this promising lithography technique for MEMS fabrication, a solid microneedle for drug delivery is simulated.
\end{abstract}

(c) 2011 Elsevier GmbH. All rights reserved.

\section{Introduction}

Even though the feature sizes in projection lithography are continuing to decrease with the forthcoming resolution enhancement techniques, the conventional contact/proximity UV lithography still plays an important role in the fabrication of various microstructures for micro-electromechanical systems (MEMS) due to their excellent performance and low processing cost. In 1994, the inclined UV lithography [1] was invented to fabricate complex 3D structures by slanting or rotating the mask and wafer system against the exposure light. Since then, the inclined/rotated UV lithography has been extensively developed and used for making many 3D structures with SU-8 thick resist [2-4], such as microchannels, out-of-plane microlenses and plano-convex lenses. However, until now there is little work [5] on simulations of the inclined/rotated UV lithography (esp. in the 3D case). Such work could greatly help lithographers to understand the underlying physical processes and to find new applications.

In this paper, a 3D model is set up to simulate the exposure process of the inclined/rotated UV lithography. Some nonlinear properties of thick resists such as light diffraction in the resist and the resist bleaching effect are included in the model. Two typical resist profiles, obtained by the inclined/rotated exposure technique, are simulated using this model in combination with a post exposure bake model for the negative-tone chemically amplified resist (CAR) and the Mack development model. As one of

\footnotetext{
* Corresponding author. Tel.: +49 0913176 1266; fax: +49 09131761212.

E-mail address: shijieliu@siom.ac.cn (S. Liu).
}

the interesting applications of the inclined/rotated UV lithography, a solid microneedle for drug delivery [6] is simulated. The impact of the exposure angle as the most flexible process parameter on the tapering angle of microneedles is analyzed.

\section{Theory}

The setup of 3D inclined/rotated UV lithography is illustrated in Fig. 1. The mask and resist coated wafer system is exposed by UV light at the incidence angle of $\theta$ in order to fabricate oblique microstructures like inclined pillars. The mask and wafer system can also be treated by several inclined exposures at different incidence angles. By using this so called multiple inclined exposures, many interesting inclined microstructures for MEMS applications have been fabricated [2]. More complicated microstructures with truncated cone shapes can be obtained by rotating the mask and wafer system against the inclined exposure light during the exposure process. The rotation speed is specified by the angular frequency $\omega$, which is defined by the change in azimuth angle $\psi$ per second.

To simulate the exposure process of inclined/rotated UV lithography, the computation of the light intensity in the resist is the first important step. The intensity distribution below the mask is determined by light diffraction at the mask, reflection and refraction at the air-resist boundary, and reflection at the resist-wafer boundary. Since the feature size of the mask for MEMS application is much larger than the wavelength of the exposure light, the light field behind the mask in the air gap can be well simulated with sufficient accuracy based on scalar diffraction methods. The further propagation of the light in the resist can be calculated by the 


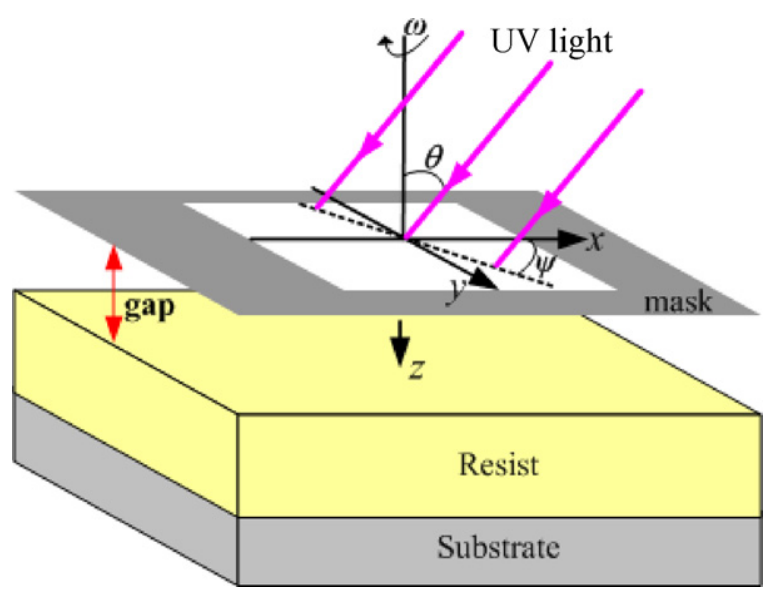

Fig. 1. Schematic diagram of the inclined/rotated UV lithography setup.

frequency domain formulation of scalar diffraction theory with the help of the transfer matrix method, which assumes that the resist is consisting of homogeneous horizontal layers. Finally, one inverse fast Fourier transform step is required to obtain the spatial distribution of the diffraction field in the resist. This calculation method is demonstrated to have fast computation speed with relatively high accuracy [7].

The SU-8 resist is a very popular CAR material to record the bulk image created by the intensity distribution of the diffracted light in MEMS fabrication. Acid is produced in the exposed region during a photochemical reaction process between resist and photons, which can be modeled by the Dill exposure model [8].

$\frac{\partial m(x, y, z, t)}{\partial t}=-C I(x, y, z, t) m(x, y, z, t)$

$h(x, y, z, t)=m(x, y, z, 0)-m(x, y, z, t)$

$n(z, t)=\left(n_{\text {unbleached }}-n_{\text {bleached }}\right) m_{\text {aver }}(z, t)+n_{\text {bleached }}$

$k(z, t)=\frac{A m_{\mathrm{aver}}(z, t)+B}{4 \pi} \lambda$

Here $m(x, y, z, t)$ is the relative photoacid generator (PAG) concentration in the resist. $I(x, y, z, t)$ and $h(x, y, z, t)$ are the light intensity and the relative acid concentration in the resist, respectively. $n(z, t)$ and $k(z, t)$ are the horizontal average values of the resist refractive index and extinction coefficient at depth position $z$, respectively. They are assumed to be linearly dependent on the horizontal average relative PAG concentration $m_{\text {aver }}(z, t) . A, B$ and $C$ are Dill parameters. $\lambda$ is the wavelength of the exposure light. $n_{\text {unbleached }}$ and $n_{\text {bleached }}$ are the refractive index of the resist before exposure and after fully exposure, respectively.

Eqs. (1)-(4) describe the exposure process including the bleaching effect inside the resist independent of an eventual inclination or rotation. The PAG concentration and the light intensity in the resist are coupled during the exposure process. They are solved with the initial condition of $m(x, y, z, 0)=1.0$ and a quasi-static approximation of $1 \mathrm{~s}$ time interval. The equations are also applicable to a multiple inclined exposure process and an inclined and rotated exposure process. When either the incident angle $\theta$ in multiple exposures or the azimuth angle $\psi$ in inclined and rotated exposure is changed, the relative PAG concentration resulting from the previous exposure will be used as the new initial value to calculate the optical constants of resist, the light intensity and the relative acid concentration inside the resist. The output of the simulation is the relative acid distribution inside the resist. Finally, the 3D resist profiles are simulated using a post exposure bake (PEB) model for the negative-tone CAR in combination with the Mack development model, which uses the relative acid concentration as the input
Table 1

SU-8 resist simulation parameters.

\begin{tabular}{ll}
\hline Parameters & Values \\
\hline Initial thickness & $5 \mu \mathrm{m}$ \\
Refractive index & $n_{\text {bleached }}=n_{\text {unbleached }}=1.6$ \\
Dill parameters & $A=-0.01 \mu \mathrm{m}^{-1}, B=0.3 \mu \mathrm{m}^{-1}, C=0.01 \mathrm{~cm}^{2} / \mathrm{mJ}$ \\
PEB parameters & Deprotection rate $=0.35 \mathrm{~s}^{-1}$, acid \\
& neutralization rate $=5 \times 10^{8} \mathrm{~s}^{-1}$, acid diffusion \\
length $=0.073 \mu \mathrm{m}$ \\
Mack parameters & $R_{\min }=0.08 \mathrm{~nm} / \mathrm{s}, R_{\max }=248 \mathrm{~nm} / \mathrm{s}, m_{\mathrm{th}}=0.33$, \\
& $n=19.8$
\end{tabular}

data. The detailed descriptions of the PEB model for CAR and the Mack development model can be found in references $[9,10]$. These methods have been implemented in the research and development lithography simulator DrLiTHO [11].

\section{Simulation results}

A mask with a $4 \mu \mathrm{m} \times 4 \mu \mathrm{m}$ contact hole is used in the simulations. The exposure is performed by $365-\mathrm{nm}$ UV light in air ambient. The other simulation parameters for SU-8 resist [12] are listed in Table 1 . Two kinds of microstructures are simulated in the following sections: inclined resist pillars based on the inclined exposure technique, and truncated cone shaped profiles based on the inclined and rotated exposure technique.

The variation of the exposure angle $\theta$ is expected to create inclined resist pillars with different slanted angles with respect to the $z$-direction. An exposure angle of $40^{\circ}$ yields an inclined resist pillar as shown in Fig. 2, where the contact exposure method is used and the exposure dose is $65 \mathrm{~mJ} / \mathrm{cm}^{2}$. The bottom $\mathrm{CD}$ of the inclined resist profile, defined as the cross section of the $3 \mathrm{D}$ resist profile in the $x z$ plane at $y=0$, is $3.46 \mu \mathrm{m}$. The slanted angle of the resist profile as shown in Fig. 2 with respect to the $z$-direction is $31.3^{\circ}$. According to Snell's law, the theoretical slanted angle for $\theta=40^{\circ}$ should be $23.7^{\circ}$. The deviation mainly results from the Fresnel diffraction of the mask image and the light absorption in the thick film resist. Nevertheless, through predicting the slanted angle with the established models, desirable inclined pillars can be easily obtained.

If the mask and wafer system is rotated against the inclined exposure light during the exposure process, resist profiles with truncated cone shapes can be obtained by overlapping the exposure steps at different azimuthal angles. When the angular frequency

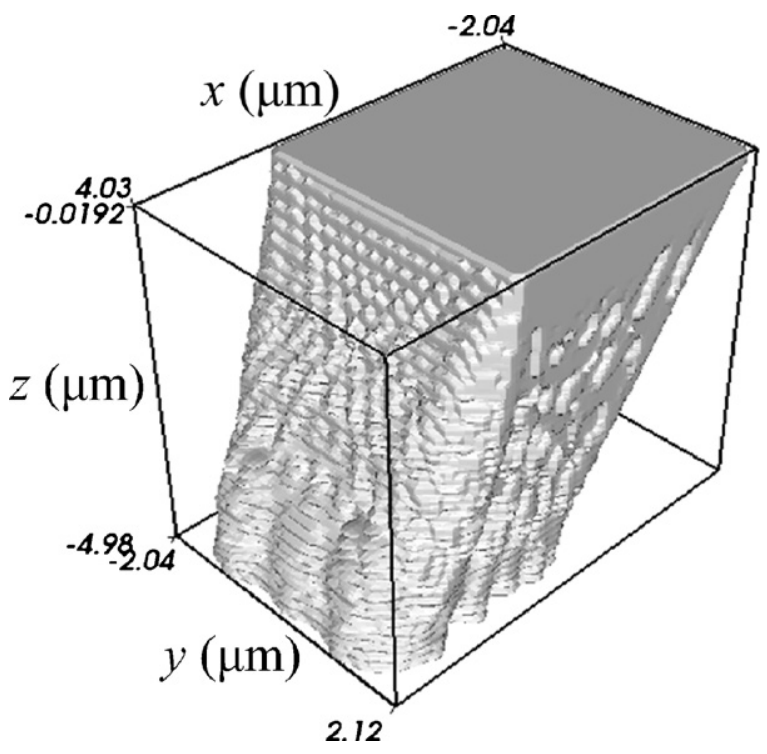

Fig. 2. Inclined pillars exposed at $\theta=40^{\circ}$. 


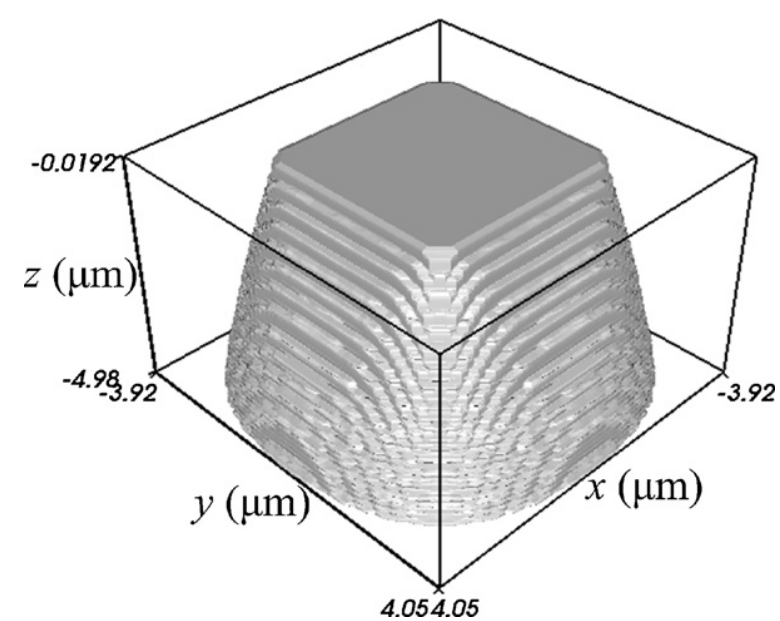

Fig. 3. Resist profile with truncated cone shape by the inclined and rotated exposure method.

$\omega$ is $10^{\circ} / \mathrm{s}$, a truncated resist profile with a smooth cone shape is obtained as shown in Fig. 3. In this simulation the exposure angle $\theta$ was $40^{\circ}$ and the exposure dose was $3600 \mathrm{~mJ} / \mathrm{cm}^{2}$. Discretization of the azimuthal angle has to be done in the simulation process. The discretization is dependent on the angular frequency. Therefore, different angular frequencies will result in some variations of the cone shaped profile with the same exposure dose. However, our simulation results show that the bottom $\mathrm{CD}$ and the tapering angle with respect to the $z$-direction of the truncated cone shaped profiles show little changes when the angular frequency is increased from $10^{\circ} / \mathrm{s}$ to $80^{\circ} / \mathrm{s}$ in steps of $10^{\circ} / \mathrm{s}$, as shown in Fig. 4 .

\section{Application to microneedles design}

Microneedles have been an attractive topic in the MEMS field. They can pierce through the outmost layer of the skin either to deliver drugs or to extract bio-liquid from the human body without pain. Polymer microneedles fabricated by UV lithography [13] have the advantages of low cost, low weight and bio-compatibility. A larger base of the polymer microneedle is required in order to improve its mechanical robustness. Therefore, a good microneedle should have a tapered microstructure with a smaller head and a larger bottom. To get this kind of resist profiles with negative SU-8 resists, Huang and Fu proposed to expose the resist from the back side of the wafer (so called backside exposure method [13]). But only a transparent substrate can be used in this method. Here, the

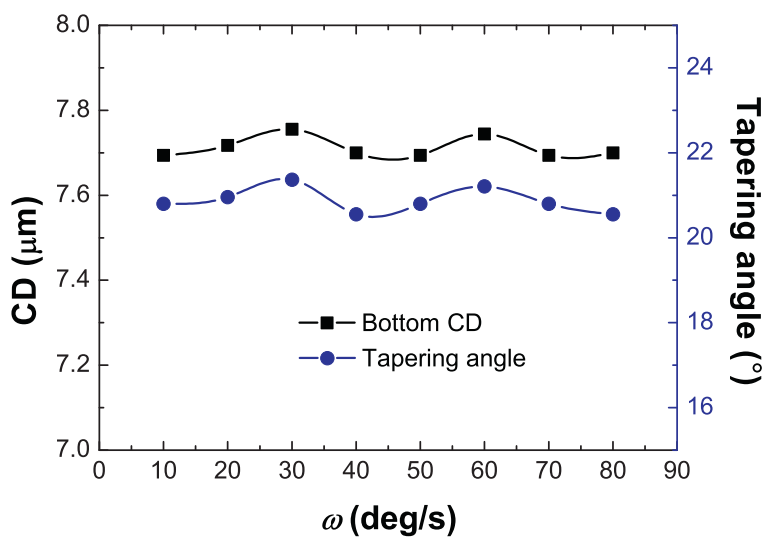

Fig. 4. $C D$ and tapering angle of truncated cone shaped resist profiles as functions of angular frequency.

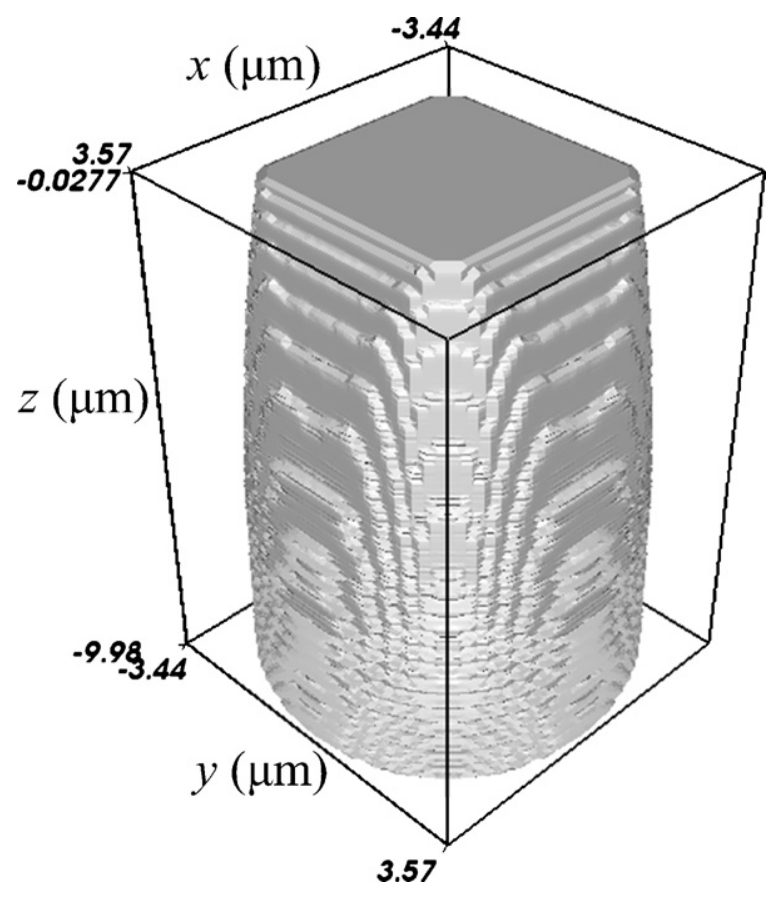

Fig. 5. Solid microneedle designed by the inclined and rotated exposure method.

inclined and rotated exposure method is applied to design SU-8 resist microneedles with no limitation on substrate material.

Fig. 5 shows a simulated design of a solid microneedle of SU8 resist by the inclined and rotated exposure method. A mask of $4 \mu \mathrm{m} \times 4 \mu \mathrm{m}$ contact hole is used. The exposure is performed by 365-nm UV light in liquid ambient with a refractive index of 1.45. The exposure angle $\theta$ and the angular frequency $\omega$ are $20^{\circ}$ and $10^{\circ} / \mathrm{s}$, respectively. The obtained resist microneedle has a top CD of $4.93 \mu \mathrm{m}$, a bottom CD of $6.74 \mu \mathrm{m}$, and a tapering angle of $5.3^{\circ}$ with respect to the $z$-direction. The tapering angle as the most critical parameter of microneedles is usually controlled by adjusting the threshold exposure dose to initiate the SU- 8 cross-linking in the backside exposure method. A more flexible method in the inclined and rotated UV lithography is to vary the exposure angle. The impact of the exposure angle on the tapering angle and CD of microneedles is shown in Fig. 6. As it can be seen, a big range of tapering angles can be obtained with this new method. The linear relationship also indicates that the exposure angle is a critical process parameter to control both the tapering angle and the $\mathrm{CD}$ of microneedles.

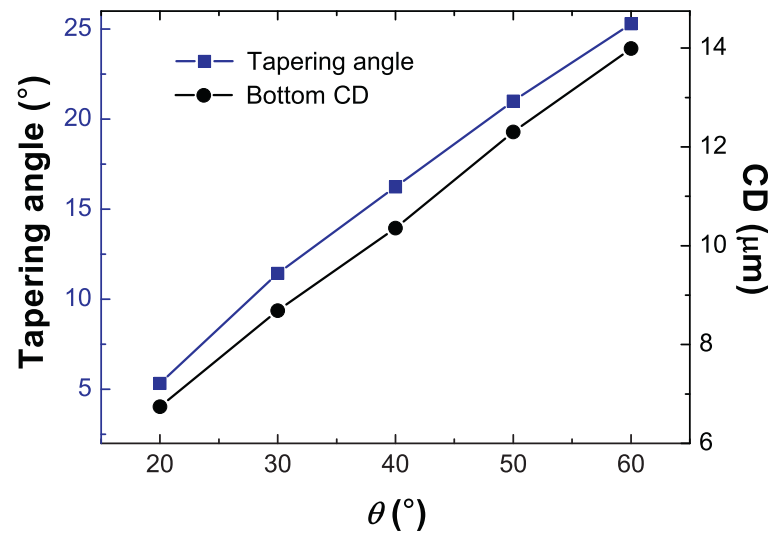

Fig. 6. Tapering angle and $C D$ of resist microneedle as functions of exposure angle. 


\section{Summary}

A 3D exposure model for the inclined/rotated UV lithography is described for SU-8 thick resists in this paper. The formation of two kinds of 3D microstructures is simulated based on the exposure model in combination with a PEB model for chemically amplified resist. The incidence angle, the exposure dose and the angular frequency in rotation exposure process are important process parameters to affect the shapes of the microstructures with this lithography technique. As one of the interesting applications of this promising lithography technique for MEMS fabrication, a solid microneedle is designed by using this technique. It is found that the exposure angle is an excellent process parameter to control the tapering angle as the most critical parameters of microneedles. Compared to the backside exposure method commonly used to fabricate microneedles, the inclined/rotated UV lithography has no limitations on substrate materials and more flexibility of tapering angle control.

\section{Acknowledgements}

The authors would like to thank the Bayerische Forschungsstiftung for funding parts of this work in the framework of the project "MALS".

\section{References}

[1] C. Beuret, G.A. Racine, J. Gobet, R. Luthier, N.F. de Rooij, Microfabrication of 3D multidirectional inclined structures by UV lithography and electroplating, Proc. IEEE Micro ElectroMech. Syst. 8 (1994) 81-85.

[2] M. Han, W. Lee, S. Lee, S.S. Lee, 3D microfabrication with inclined/rotated UV lithography, Sens. Actuators A 111 (2004) 14-20.

[3] Z. Ling, K. Lian, SU-8 3D microoptic components fabricated by inclined UV lithography in water, Microsyst. Technol. 13 (2007) 245-251.

[4] H. Sato, D. Yagyu, S. Ito, S. Shoji, Improved inclined multi-lithography using water as exposure medium and its 3D mixing microchannel application, Sens. Actuators A 128 (2006) 183-190.

[5] Z. Zhu, Z. Zhou, Q. Huang, W. Li, Modeling, simulation and experimental verification of inclined UV lithography for SU-8 negative thick photoresists, J. Micromech. Microeng. 18 (2008) 125017.

[6] K. Kim, D. Park, H. Lu, W. Che, K. Kim, J. Lee, C. Ahn, A tapered hollow metallic microneedle array using backside exposure of SU-8, J. Micromech. Microeng. 14 (2007) 597-603.

[7] B. Meliorisz, A. Erdmann, Simulation of proximity printing, J. Micromech. Microeng. 6 (2007) 023006.

[8] F. Dill, W. Hornberger, P. Hauge, J. Shaw, Characterization of positive photoresist, IEEE Trans. Electron. Dev. 7 (1975) 445-452.

[9] A. Erdmann, W. Henke, S. Robertson, E. Richter, B. Tollkühn, W. Hoppe, Comparison of simulation approaches for chemically amplified resists, Proc. SPIE 4404 (2001) 99-110.

[10] C. Mack, Development of positive photoresists, J. Electrochem. Soc. 134 (1987) $148-152$.

[11] T. Fühner, T. Schnattinger, G. Ardelean, A. Erdmann, Dr. LiTHO - a development and research lithography simulator, Proc. SPIE 6520 (2007) 65203F.

[12] Y. Sensu, A. Sekiguchi, S. Mori, N. Honda, Profile simulation of SU-8 thick film resist, Proc. SPIE 5753 (2005) 1170-1185.

[13] H. Huang, C. Fu, Different fabrication methods of out of plane polymer hollow needle arrays and their variations, J. Micromech. Microeng. 17 (2007) 393-402. 